ORIGINAL ARTICLE

\title{
Running exercises improve the strength of a partially ruptured Achilles tendon
}

\author{
E K N See, G Y F Ng, C O Y Ng, D T C Fung
}

Br J Sports Med 2004;38:597-600. doi: 10.1136/bjsm.2003.007120

See end of article for authors' affiliations

......................

Correspondence to: Professor Gabriel Ng, Department of

Rehabilitation Sciences, The Hong Kong

Polytechnic University,

Hung Hom, Kowloon,

Hong Kong;

rsgng@polyu.edu.hk

Accepted 27 June 2003

\begin{abstract}
Objectives: To examine the effects of running and swimming exercises on the functional performance and mechanical strength of a recovering Achilles tendon.

Methods: 30 Sprague-Dawley rats had surgical transection of their right medial Achilles tendon. The rats were divided into running $(n=11)$, swimming $(n=10)$, and control $(n=9)$ groups. The running and swimming groups were given daily exercise training, starting from the fifth day after the injury; the control group did not exercise throughout the period of the experiment. An Achilles functional index (AFI) was recorded before the operation and on the third, 10th, and 30th days after the operation. On the 30th day, the rats were killed and their Achilles tendons harvested for biomechanical testing of load relaxation properties, stiffness, and ultimate tensile strength (UTS). The AFI data were analysed by two way analysis of variance; load relaxation, stiffness, and UTS data were analysed by multivariate analysis, with $\alpha$ at 0.05 .

Results: The UTS of the running group was higher than in the control group $(p=0.015)$, while there was no significant difference between the swimming and control groups $(p=0.228)$. Differences in stiffness and load relaxation were non-significant ( $p=0.823$ and 0.633 , respectively). The AFI results did not differ among the three groups $(p=0.242)$.

Conclusions: Running exercises can improve the strength of partially ruptured Achilles tendons at 30 days after injury.
\end{abstract}

$\mathrm{T}$ ndons heal slowly after injury, and the rupture of Achilles tendons usually means weeks of immobilisation with or without surgical repair. ${ }^{1}$ However, immobilisation of the ankle joint, even for a short time, has an adverse effect on function, biochemical properties, and biomechanical strength of both normal and injured Achilles tendons in the rat. ${ }^{2}{ }^{3}$ Early functional loading and controlled movements have been reported to augment the strength of healing Achilles tendons without causing them to re-rupture. ${ }^{4-6}$ Enwemeka et al reported that rats starting weight bearing exercises five days after Achilles tendon injuries had a significantly higher breaking strength in the tendons than control animals or rats that began exercising two days after the injury. ${ }^{6}$ The same investigators ${ }^{5}$ also reported that functional loading beginning five days after tenotomy led to a significant increase in the tensile strength and energy absorption capacity of the Achilles tendons of rabbits. Date ${ }^{4}$ studied the effect of loading on the ultrastructure of healing tendons and showed that the diameter of the collagen fibres increased, suggesting a more rapid collagen maturation process.

It has been found that in normal tendons the concentration of collagen, the size of collagen fibrils, and fibril density increase after exercise. ${ }^{7}$ These ultrastructural morphological changes are manifested by mechanical improvement in the tendons, and it has been well documented that the ultimate tensile strength of normal tendons increases with swimming and running. ${ }^{89}$ Simonsen et al showed that the ultimate tensile strength of tendons in swimming trained animals was significantly higher than in their strength trained counterparts. ${ }^{9}$ Tendons seem to respond to endurance exercises but not to high loading strength training. These effects of exercise training might also be manifested in tendons under repair.

However, only a few studies have examined the effects of exercise on healing ligaments and tendons. Studies have shown that the effects of training on the tensile strength and biochemical properties of transected medial collateral ligament were significant with enforced exercises, ${ }^{10}{ }^{11}$ but the same effect was not demonstrated in tendons during healing. Murrel et al reported that 15 minutes of daily swimming exercise had no effect on the biomechanical properties of healing tendons as compared with a non-exercised group. ${ }^{12}$ However, the non-significant results of Murrel's study did not necessarily infer that exercise training was unable to improve tendon strength. The negative results could have reflected other factors such as the mode of injury, as the researchers had used a complete sectioning injury model of all plantar flexors. This had caused the cut ends of the tendons to retract, thus rendering healing difficult. Furthermore, the duration of the exercise or the type of loading might not have been large enough to lead to any effects. The exercises could have begun too early, as the animals were put to swim immediately after the injury when inflammation was still intense, which might have caused the inflammation to flare up, leading to overscarring and abnormal tissue strength.

In view of the above, it is necessary to conduct a study to simulate the conditions of partial tendon rupture, so that the cut ends are not retracted and healing is not impeded..$^{13}$ The exercises should also be delayed for a few days to avoid aggravating the acute inflammation. ${ }^{14}$ To date, no study has been reported comparing the effects of different types of exercise on a partially injured tendon. Furthermore, the viscoelastic properties of repairing tendons have not been investigated; thus their behaviour under sustained loading is not known. We therefore conducted this study to examine the effects of exercise on the biomechanical and functional properties of the partially injured Achilles tendons of rats,

Abbreviations: AFI, Achilles functional index; UTS, ultimate tensile strength 
and to compare the effects of weight bearing and nonweight-bearing exercises on tendons that are healing.

\section{METHODS \\ Subjects}

Thirty Sprague-Dawley rats aged three months were used. Their average weight at the time of surgery was $472 \mathrm{~g}$ (range 375 to 575). The animal subjects ethics review committee of the Hong Kong Polytechnic University reviewed and approved the procedures of this study.

Each rat had complete transection of the medial part of the right Achilles tendon, leaving the lateral tendon intact as an internal splint to prevent the cut ends from retracting. They were randomly divided into three groups: group 1 , no exercise (control); group 2, running exercises; group 3, swimming exercises.

\section{Surgical procedures}

All surgical procedures were carried out under general anaesthesia with an intraperitoneal injection of a mixture of $100 \mathrm{mg} / \mathrm{kg}$ ketamine and $20 \mathrm{mg} / \mathrm{kg}$ xylazine (both from Alfasan International, Woerden, Netherlands). The dosages were calculated according to the ratio of $0.6 \mathrm{mg}$ ketamine and $0.3 \mathrm{mg}$ xylazine per $100 \mathrm{~g}$ body weight. The skin on the anterior aspect of the right calf was shaved, incised, and retracted to expose the Achilles tendon. The tendon of the medial gastrocnemius was identified and separated from the lateral tendon with a probe; the medial tendon was then transected with a scalpel at its mid point. The lateral tendon and plantaris were left intact to simulate the case of a partial tendon rupture, so as to prevent the ruptured ends from retracting. ${ }^{13}$ The skin wound on the anterior calf was closed by a suture. After the operation, the animals were divided into groups of five, each group being placed in a $20 \times 24 \times 40$ $\mathrm{cm}$ cage. No cast immobilisation was applied, and the rats were allowed to move unhindered inside the cages. All the animals were kept under a 12 hour light/dark cycle, with temperatures regulated at about $20^{\circ} \mathrm{C}$ and relative humidity at $50 \%$. Tap water and food were given ad libitum during the whole study.

\section{Exercise programme}

The timing of exercise followed a previous report. ${ }^{5}$ On the fifth day after the operation, the two exercise groups began their respective exercise programmes. All the rats in the running group ran on a treadmill at an initial speed of $4.0 \mathrm{~m} / \mathrm{min}$ for the first two days of the exercise. This speed was used in order to acquaint the rats with the running exercise. The speed was gradually increased to $12 \mathrm{~m} / \mathrm{min}$ throughout the training period. The rats in the swimming group swam in a rectangular water tank measuring $39 \times 70 \times 40 \mathrm{~cm}$ filled with water at $25^{\circ} \mathrm{C}$. The water level was adjusted so that the rats could not touch the floor of the tank with their legs.

All the animals in both exercise groups were exercised for six days a week, starting from the fifth day after the operation until the 30th. The duration of the exercises was progressively increased from 15 minutes in the first week to 17 minutes in the second week, and then to 19 minutes for the remainder of the time.

\section{Measurements}

\section{Functional testing}

Functional testing of the Achilles tendon was done in all animals preoperatively and then on postoperative days 3,10 , and 30. We used the Achilles functional index (AFI), reported in the study by Murrel et al. ${ }^{15}$ This test has proved reliable and sensitive to healing time, and shows excellent correlation with the properties of load to failure. ${ }^{16}$ The rats were tested in a walkway $10 \mathrm{~cm}$ wide and $60 \mathrm{~cm}$ long with a white paper underlay. Their hind paws were dipped in blue paint, and the rat was made to walk down the walkway, leaving blue footprints on the white underlay surface. The papers were coded by the animal's number and stored to be measured later in a single blind fashion.

Measurements were taken from the middle four footprints marked on the paper. The maximum length of a foot print is defined as the print length factor (PLF), the distance between the first and fifth toes is the toe spreading factor (TSF), and the distance between the second and fourth toes is the intermediary test factor (ITF). These factors were measured and the AFI was calculated according to an established formula ${ }^{15}$ :

$$
\mathrm{AFI}=74(\mathrm{PLF})+161(\mathrm{TSF})+48(\mathrm{ITF})-5 .
$$

\section{Biomechanical testing}

The rats were killed on the 30th day after the operation by an overdose injection of anaesthesia. Both lower limbs were harvested by disarticulating them at the hip joints. The specimens were then sealed in a plastic bag and stored at $-40^{\circ} \mathrm{C}$ for later testing.

At least six hours before testing, the specimens were retrieved from the freezer and allowed to thaw inside the plastic bag at room temperature. The specimens were dissected to remove all soft tissues, leaving the intramuscular tendinous fibres, Achilles tendon, and calcaneus intact. The intramuscular tendinous fibres were then secured between two strips of plastic with quick setting superglue. Care was taken to prevent the glue from running down the tendon, and the specimens were kept moist with normal saline during the whole testing procedure.

The glued strip and the calcaneus were then mounted on the cross heads of a material testing machine (MTS Synergie 200, MTS Systems, Minnesota, Minneapolis, USA). An extensometer (MTS model 634: 12F-24, MTS Systems) was attached to the margin of the cross heads for measuring the local strain in the tendon. The room temperature was controlled at $25^{\circ} \mathrm{C}$ throughout testing.

\section{Testing for viscoelasticity}

Each specimen mounted on the material testing machine was preconditioned with 10 oscillation cycles of $2.5 \%$ strain at a rate of $10 \mathrm{~mm} / \mathrm{min}$ to eliminate the effect of deep freezing on the tissue. ${ }^{17} 18$ After preconditioning, the specimen was elongated to $2.5 \%$ strain and maintained for five minutes. ${ }^{19}$ The loads were recorded throughout the test at a sampling rate of $5 \mathrm{~Hz}$. To quantify the load relaxation property, the difference between the initial and final load was expressed as a percentage of the initial load.

\section{Testing for ultimate tensile strength}

After testing for viscoelasticity, the specimen was unloaded and left for five minutes on the machine to let the tendon return to its original length. It was then subjected to the failure test at a loading rate of $500 \mathrm{~mm} / \mathrm{min}$ until failure. ${ }^{19}$ Load and displacement data were recorded at a sampling rate of $50 \mathrm{~Hz}$. The maximum load recorded represents the ultimate tensile strength (UTS), and the gradient in the linear portion immediately after the toe region of the load displacement curve represents the stiffness value.

\section{Statistical analysis}

The within-subject reliability of the AFI measurements was established with intraclass correlation coefficients, ${ }^{13}$ with two measurements of the preoperative recording. A two way repeated measures analysis of variance (ANOVA) was used to analyse the AFI data. A multivariate analysis of variance 
(MANOVA) was used to analyse the load relaxation, UTS, and stiffness data. The independent variable was the grouping. The significance level was set at 0.05 for all the tests.

\section{RESULTS}

On visual examination, the Achilles tendon in all groups showed signs of repair, with thick fibrous scar formation around the injury sites. The mean ICC for the preoperative AFI measurements was 0.97 , which indicates good reliability for the AFI measurements. However, the result of the AFI was not significant among the three groups $(p=0.242)$. The AFI values of all groups fell from the values recorded on the preoperative day to reach a low on postoperative day 3, and before gradually improving on days 10 and 30 (fig 1).

The results of load relaxation and stiffness were not significantly different among the groups $(\mathrm{p}=0.633$ and $\mathrm{p}=0.823$, respectively). However, the difference in the UTS was significant $(p=0.049)$. Post hoc analysis showed that the UTS of the running group was greater than in the control group $(p=0.015)$, while the value in the swimming group did not differ from that in the control group $(p=0.228)$ (table 1).

\section{DISCUSSION}

In this study we found that running exercises can promote the recovery of partially transected Achilles tendon in rats. Previous studies have shown that in the Achilles tendon of healing rabbits, inflammation subsides within seven days after injury, and fibroplasia and fibrillogenesis begin at about five days after the injury, with fibroblasts and newly synthesised collagen scattering in the tissue. ${ }^{14}{ }^{20}$ From 12 days onward, collagen synthesis occurs concurrently with matrix remodelling, characterised by the aggregation of the collagen fibrils into bundles that are easily observable by day $21 .{ }^{1420}$

The high UTS of our running group could reflect accelerated fibroblast proliferation, fibrillogenesis, and matrix remodelling as a result of the exercise loading on the tendon. In previous studies it has been shown that mechanical loading can enhance the parallel alignment and polymerisation of the collagen fibrils and the production of glycosaminoglycans. ${ }^{21-23}$ Becker and Diegelmann ${ }^{21}$ reported that the fibroblasts of tendons under loading were better aligned than those of tendons without loading. On another study it was found that mechanical loading facilitated the formation of intracytoplasmic collagen fibrils, a phenomenon associated with the rapid synthesis of collagen, and that this phenomenon was not shown in tendons immobilised by casts. ${ }^{22}$ Furthermore, the mechanical forces of weight bearing

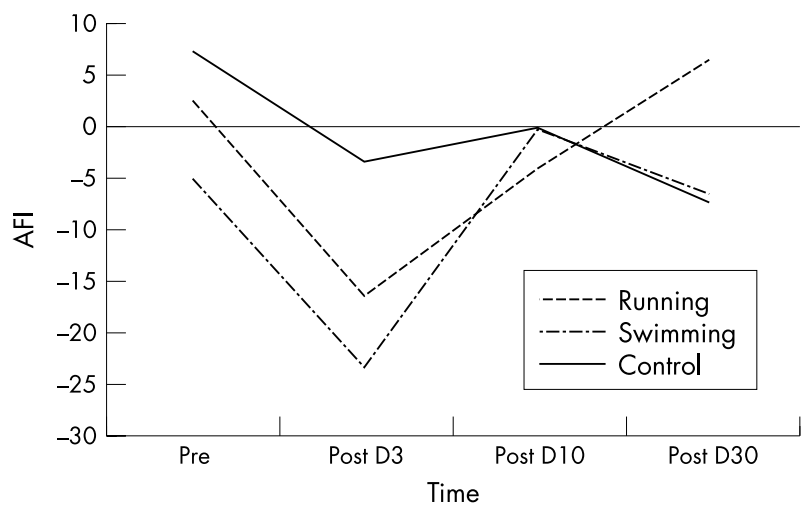

Figure 1 The pattern of change in Achilles functional index (AFI) of the control, running, and swimming groups on the preoperative day (Pre), postoperative day 3 (Post D3), day 10 (Post D10), and day 30 (Post D30).
Table 1 Ultimate tensile strength (UTS), stiffness, and per cent load relaxation in the three groups

\begin{tabular}{lllll}
\hline & $\begin{array}{l}\text { Control } \\
(\mathbf{n}=9)\end{array}$ & $\begin{array}{l}\text { Running } \\
(\mathbf{n}=11)\end{array}$ & $\begin{array}{l}\text { Swimming } \\
(\mathbf{n}=10)\end{array}$ & p Value \\
\hline UTS (N) & $61.7 \dagger(12.6)$ & $73.8+(9.0)$ & $67.6(9.7)$ & $0.049^{*}$ \\
Stiffness (N/mm) & $71.5(18.0)$ & $76.5(15.9)$ & $72.2(24.3)$ & 0.823 \\
Load relaxation (\%) & $38.4(12.6)$ & $37.7(9.0)$ & $40.5(9.7)$ & 0.633 \\
\hline Values are mean (SD). The p values are derived from analysis of variance \\
(ANOVA). \\
*Significant difference in the ANOVA result. \\
†Significant difference with post-hoc linear contrasts for between-group \\
comparisons.
\end{tabular}

and locomotion may augment the tensile strength of the Achilles tendon. ${ }^{56}$ The greater strength in tendons that had been subjected to exercise could reflect accelerated fibroblast proliferation, fibrillogenesis, and matrix remodelling, because it is the collagen content and arrangement of the fibrils that determines strength. ${ }^{23} 24$

Our finding that the load relaxation and stiffness of the running group did not differ from the control group could have resulted from the low statistical power $(<0.2)$ of our comparisons for both these variables. Furthermore, load relaxation is a viscoelastic property, whereas stiffness and UTS are structural properties. It has been suggested that viscoelasticity is determined by the interaction between small sized collagen fibrils, and structural properties by large sized collagen fibrils. ${ }^{25}$ The non-significant difference in load relaxation among the three groups could indicate that exercise has no effect on the ultrastructural morphological profile of the small collagen fibrils. This will need to be explored further with ultrastructural morphological studies. As for stiffness and UTS, the former is a measurement at submaximal loading while the latter is a measurement of the maximum strength. Thirty days after injury, the tendons might have recovered to such an extent that the submaximal property was normal, so that no difference was found in stiffness measurements.

As both stiffness and UTS are derived from the same load deformation curve, differences in UTS but not in stiffness among groups would imply a larger area under the curve, and thus a higher energy absorption capacity in the running group. This may be explained by the structural morphology. Textural birefringence measurements in the tendons of exercised animals have shown that the process of adaptation to a new level of functional stimulation results in the reorganisation of the extracellular matrix elements. At the macromolecular level, this corresponds to better alignments of the collagen bundles with more organised intermolecular packing orders than those of the controls. ${ }^{23}$ Becker and Diegelmann $^{21}$ showed that fibroblasts were stretched out parallel to the direction of tension compared with the random orientation of control tendons without tension. Exercise might have induced the fibres to reorientate to the extent that they became able to withstand higher tensile forces and absorb more energy before breakage.

The results of our swimming group are in line with the findings of Murrel et al, ${ }^{12}$ who also reported no significant benefits of swimming exercises for the strength of repairing tendons. This could reflect the mechanical demands of the swimming group. Although swimming is an active exercise, the loading in the Achilles tendon is small because of its non-weight-bearing nature. The small loading may not have been enough to stimulate fibroblast proliferation and fibrillogenesis.

Comparing our study with that of Burroughs and Dahners, ${ }^{10}$ the exercise intensity for the swimming group was similar between the two studies, but the findings were 


\section{Take home message}

- Exercise has been reported to improve the strength of repairing tendons. However, it is not known whether exercise may benefit tendons with partial injury, and if it does, what type of loading is of greatest benefit. This study shows that running exercise can significantly strengthen a partially injured Achilles tendon, but this effect was not found with swimming exercise.

different. Burroughs and Dahners found that enforced swimming exercises had a beneficial effect on repairing medial collateral ligament strength, but we did not find such an improvement in the Achilles tendon. This may be explained by the action of swimming, which involves substantial movements in the knee joints and relatively little movement in the ankles. The movements in the knee could lead to loading on the joint structures, thus facilitating fibroblast activity and the collagen remodelling process in the medial collateral ligament, whereas the limited movement in the ankle may deprive the Achilles tendon of sufficient loading.

The fact that functional behaviour was not significantly different among the three groups could be because this test was not sensitive enough to detect a difference. Best et al reported that AFI correlated with the biomechanical variables and was sensitive to changes during the healing process of a total tendon transection injury model. ${ }^{16}$ In our study, the tendon was partially transected and the injury was less severe. Thus the functional difference between each group during the healing process would not be as great as that seen in a complete transection injury model. Because of this, the AFI might not be sensitive enough to detect these small differences. According to the timing of healing, an injured tendon will take about three weeks to regain the approximate strength and functioning of a normal tendon. ${ }^{26}$ The finding that UTS, but not AFI, was significantly different could be because UTS is a measure of maximum strength while AFI measures the function under a submaximal level of loading. At four weeks, the tendons might have attained enough strength to withstand a normal functional loading during walking, but there was still a deficit compared with the maximum strength of the control group.

Care must be taken when applying the result of this study to humans. Beside the fundamental differences between rats and humans-such as the metabolism, anatomy, and movement pattern - the tendon injuries in this study were induced under controlled conditions with a scalpel so as to standardise the mechanism of injury. This does not simulate the clinical situation because tendons are usually injured by uncontrolled tensile loading. Another difference is the predisposing factor of this injury, as rupture of the Achilles tendon usually occurs in middle aged individuals who may have vascular impairment and degenerative changes in the tendon. ${ }^{27}$ Thus the tendons may already show pathological changes even before the rupture, and these could hinder the healing process.

There do not seem to have been any studies investigating the effect of exercise on a partially transected Achilles tendon. Our results have shown that running exercises can improve the strength of a partially transected tendon in which the two ends are not retracted. Although the timing of healing in rats may differ from that in humans, this finding will serve as a guide for future studies on the clinical treatment approach, such that early exercise loading should be considered for tendon healing when the rupture ends are in close approximation, either in cases of partial rupture or after surgical repair for total rupture.

\section{ACKNOWLEDGEMENTS}

We thank Dr Mason Leung for his technical advice and the Department of Rehabilitation Sciences of The Hong Kong Polytechnic University for its financial support of the study.

\section{Authors' affiliations}

E K N See, G Y F Ng, C O Y Ng, D T C Fung, Department of Rehabilitation Sciences, The Hong Kong Polytechnic University, Kowloon, Hong Kong

\section{REFERENCES}

1 Willis CA, Washburn S, Caiozzo V, et al. Achilles tendon rupture. A review of the literature comparing surgical versus nonsurgical treatment. Clin Orthop 1986;207:156-63.

2 Kvist $M$, Hurme T, Kannus $P$, et al. Vascular density at the myotendinous junction of the rat gastrocnemius muscle after immobilization and remobilization. Am J Phys Med Rehabil 1995;23:359-64.

3 Murrell GAC, Lilly EG, et al. Effects of immobilization Achilles tendon healing in a rat model. J Ortho Res 1994;12:582-91.

4 Date T. The influence of exercise in the healing of rabbits Achilles tendon. J Jpn Orthop Ass 1986;60:449-54.

5 Enwemeka CS, Spielholz NI, et al. The effect of early functional activities on experimentally tenotomized Achilles tendons in rats. Am J Phys Med Rehabil 1989;67:264-9.

6 Enwemeka CS. Functional loading augments the initial tensile strength and energy absorption capacity of regenerating rabbit Achilles tendons. Am J Phys Med Rehabil 1992;71:31-8.

7 Enwemeka CS, Maxwell LC, Fernandes G. Ultrastructural morphometry of matrical changes induced by exercise and food restriction in the rat calcaneal tendon. Tissue Cell 1992;24:499-510.

8 Buchanan $\mathrm{Cl}$, Marsh L. Effects of long-term exercise on the biomechanical properties of the Achilles tendon of guinea fowl. J Appl Physiol $2001 ; 90: 164-71$.

9 Simonsen EB, Klitgaard H, Bojsen-Moller F. The influence of strength training, swim training and ageing on the Achilles tendon and $\mathrm{m}$. soleus of the rat. J Sports Sci 1995;13:291-5.

10 Burroughs $\mathbf{P}$, Dahners LE. The effect of enforced exercise on the healing of ligament injuries. Am J Sports Med 1990;18:376-8.

11 Vailas AC, Tipton CM, Matthes RD, et al. Physical activity and its influence on the repair process of medial collateral ligaments. Connect Tissue Res 1981;9:25-31.

12 Murrel GAC, Jang D, Deng XH, et al. Effects of exercise on Achilles tendon healing in a rat model. Foot Ankle 1998;19:598-603.

$13 \mathrm{Ng}$ GYF, Oakes BW, McLean LD, et al. The long-term biomechanical and viscoelastic performance of repairing anterior cruciate ligament after hemitransection injury in a goat model. Am J Sports Med 1996:24:109-17.

14 Enwemeka CS. Inflammation, Cellularity, and fibrillogenesis in regenerating tendon: Implications for tendon rehabilitation. Phys Ther 1989;69:816-25.

15 Murrell GAC, Lilly EG, Davies H, et al. The Achilles functional index. J Orthop Res 1992;10:398-404.

16 Best TM, Collins A, Lilly EG, et al. Achilles tendon healing: a correlation between functional and mechanical performance in the rat. J Orthop Res 1993; 11:897-906

17 Woo SL, Orlando CA, Camp JF, et al. Effects of postmortem storage by freezing on ligament tensile behaviour. J Biomech 1986;19:399-404.

$18 \mathrm{Ng} \mathrm{GYF}$, Oakes BW, Deacon O, et al. Biomechanics of patellar tendon autograft for reconstruction of the anterior cruciate ligament in the goat: threeyear study. J Orthop Res 1995;13:602-8.

19 Fung DTC, Ng GYF, Leung MCP, et al. Tay. Therapeutic low energy laser improves the mechanical strength of repairing medial collateral ligament. Lasers Surg Med 2002;31:91-6.

20 Postacchini F, Accinni L, Natali PG, et al. Regeneration of rabbit calcaneal tendon: a morphological and immunochemical study. Cell Tiss Res 1978; 195:81-97.

21 Becker H, Diegelmann RF. The influence of tension on intrinsic tendon fibroplasias. Orthop Rev 1984;13:65-71.

22 Enwemeka CS. Membrane-bound intracellular collagen fibrils in fibroblasts and myofibroblasts of regenerating rabbit calcaneal tendons. Tissue Cell 1991;23:173-90.

23 Vilarta R, Vidal B. Anisotropic and biomechanical properties of tendons modified by exercise and denervation: aggregation and macromolecular order in collagen bundles. Matrix 1989;9:55-61

24 Vogel HG. Correlation between tensile strength and collagen content in rat skin. Effect of age and cortisol treatment. Connect Tissue Res 1974;2:177-82.

25 Parry DAD, Barnes GRG, Craig AS. A comparison of the size distribution of collagen fibrils in connective tissues as a function of age and a possible relation between fibril size distribution and mechanical properties. Proc $R$ Soc Lond B 1978;203:305-21.

26 Sandberg N, Zederfeldt B. The tensile strength of healing wounds and collagen formation in rats and rabbits. Acta Chir Scand 1963;126:187-96.

27 Carr AJ, Norris SH. The blood supply of the calcaneal tendon. J Bone Joint Surg Br 1989;71B:100-1. 